\title{
Calibration Method for IATS and Application in Multi-Target Monitoring using Coded Targets
}

\author{
Y. Zhou ${ }^{1,2}$, A. Wagner ${ }^{2}$, T. Wunderlich ${ }^{2}$, P. Wasmeier ${ }^{2}$ \\ 1. College of Surveying and Geo-Informatics, Tongji University, Shanghai, China \\ 2. Chair of Geodesy, Technische Universität München, Munich, Germany
}

\begin{abstract}
The technique of Image Assisted Total Stations (IATS) has been studied for over ten years and is composed of two major parts: one is the calibration procedure which combines the relationship between the camera system and the theodolite system; the other is the automatic target detection on the image by various methods of photogrammetry or computer vision. Several calibration methods have been developed, mostly using prototypes with an add-on camera rigidly mounted on the total station. However, these prototypes are not commercially available. This paper proposes a calibration method based on Leica MS50 which has two built-in cameras each with a resolution of $2560 \times 1920 p x$ : an overview camera and a telescope (on-axis) camera. Our work in this paper is based on the on-axis camera which uses the 30-times magnification of the telescope. The calibration consists of only 5 parameters to estimate. We use coded targets, which are common tools in photogrammetry for orientation, to detect different targets in IATS images. We test and verify the efficiency and stability of this method using multi-target monitoring.
\end{abstract}

Keywords. Image Assisted Total Station, Coded Targets, Calibration, Multi-Target Monitoring

\section{Introduction}

Image Assisted Total Stations are a promising solution for replacing human vision in some traditional surveying applications. It combines all the advantages of total stations and cameras such as high automation, human error elimination, etc.

Wagner (2016) describes several modern total stations which are equipped with at least one (overview) camera. Among them, the MS50, modern total station produced by Leica Geosystems, is one of those which is also equipped with a telescope camera and has a different field-of-view (FoV). The calibration method in this paper is developed for the built-in telescope camera which has a small FoV. The maximum resolution of the telescope camera is $2560 \times 1920 p x$ and the camera axis is parallel with the collimation axis. One of the principal problems using IATS is establishing the relationship between image points and the corresponding angular readings in the theodolite coordinate system. Most of the existing calibration methods are tested on different prototypes, e.g. Walser (2004), Knoblach (2009), Bürki et al. (2010).

Bürki et al. (2010) provide an overview of traditional active Automated Target Recognition (ATR) technique and its limitations, while the adaptation of an appropriate CCD camera can help to solve some of these problems. Automatic target detection based on images improves the efficiency of IATS applications through ellipse detection, cross line detection, template matching, etc. For actual applications, coded targets (CTs) are probably the best method because by CTs, both the pixel coordinate value and the ID of the target on the image can be obtained at the same time. CTs are one of the basic tools in photogrammetry and are primarily used for initial exterior orientation determination (Hattori et al. 2002). There are two main types of CTs, concentric rings and dot distribution (Hattori et al. 2000). We choose the first one because it's more robust in detection. Based on open-source libraries (Bradski 2000), CTs are applied to automatically detect the pixel coordinate of the target on the image.

\section{Mathematical Model of Calibration}

Huang and Harley (1989) originally proposed a camera calibration method without a real control array. It is suitable not only for the built-in telescope camera, but also for other cameras which are rigidly installed at the telescope. The accuracy of distance

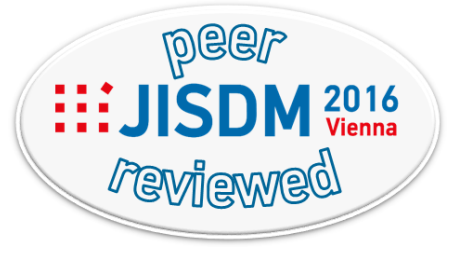


measurement is relatively lower than that of the angular measurement for most of the total stations in close range. It demands more stringent requirements for the distance measurement if we want to yield higher accuracy of $3 \mathrm{D}$ coordinates. Thus, distance measurement is not involved in this method and we only concentrate on the 2D transformation relationship between angular measurement and image coordinate.

The pixel coordinate system is a left handed (LH) coordinate system. The origin is the left top point on the image. We define the image coordinate system also as a LH coordinate system. We define the virtual principal point $\left(x_{0}, y_{0}\right)$ as the origin $O_{-} i m g$ in the image coordinate system, and this point is always fixed. The relationship between the two coordinate sets is as shown in Fig. 1, and we have:

$$
\begin{aligned}
& x I=y_{0}-y P \\
& y I=-x_{0}+x P
\end{aligned}
$$

where $x P, y P$ are pixel coordinates, $x I, y I$ are image coordinates.

Distortion occurs inevitably in lens systems, especially in wide FoV lenses (Devernay and Faugeras 2001, Tsai 1987). But in our case where the $\mathrm{FoV}$ is 1.7 gon diagonal, the distortion error is much smaller. Thus, we only consider one radial distortion parameter. The corrected image coordinate is:

$$
\begin{aligned}
& x I_{-} c=x I+x I \cdot K_{1} \cdot r^{2} \\
& y I_{-} c=y I+y I \cdot K_{1} \cdot r^{2}
\end{aligned}
$$

where $x I \_c, y I \_c$ are corrected image coordinates, $r^{2}=\left(x I^{2}+y I^{2}\right)$ and $K_{l}$ is the radial distortion parameter.

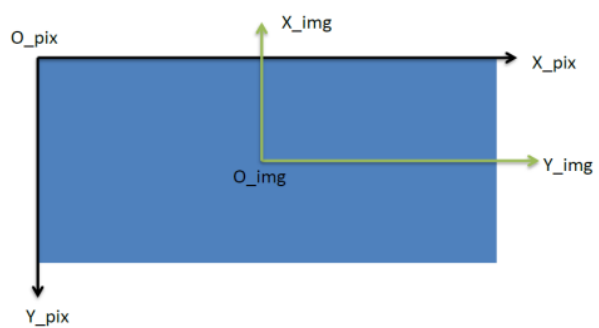

Fig. 1 Relationship between pixel coordinate system and image coordinate system.
Supposing there is a virtual plane which is perpendicular to the collimation axis, we also define the 3D telescope LH coordinate system in which the $\mathrm{Z}$ axis is opposite to the pointing direction and $\mathrm{Y}$ axis is the horizontal axis. The $\mathrm{X}$ and $\mathrm{Y}$ axis in this virtual plane coordinate system are parallel to the $X$ and $\mathrm{Y}$ axis in the telescope coordinate system, so the $\mathrm{X}$ and $\mathrm{Y}$ coordinates on the virtual plane are the same with those in the telescope coordinate system.

According to Leica Geosystems AG (2013), the telescope camera axis is parallel to the collimation axis in MS50. In fact, these two axes can't be ideally parallel because of manufacture error. Thus, the image plane and the virtual plane will intersect at line A-B as shown in Fig. 2. It is obvious that there will be a geometric deformation if we vertically project a shape from the image plane to the virtual plane. Along S2 direction, the scale value is zero, but along $\mathrm{S} 1$ direction, the scale value is maximal. Actually the scale factor along S1 direction can be decomposed to both axes in the image plane coordinate system, which are $S_{x}$ and $S_{y}$ respectively. This is the reason why we can still consider that the collimation axis and the camera axis are parallel, because the difference can be compensated by an affine transformation.

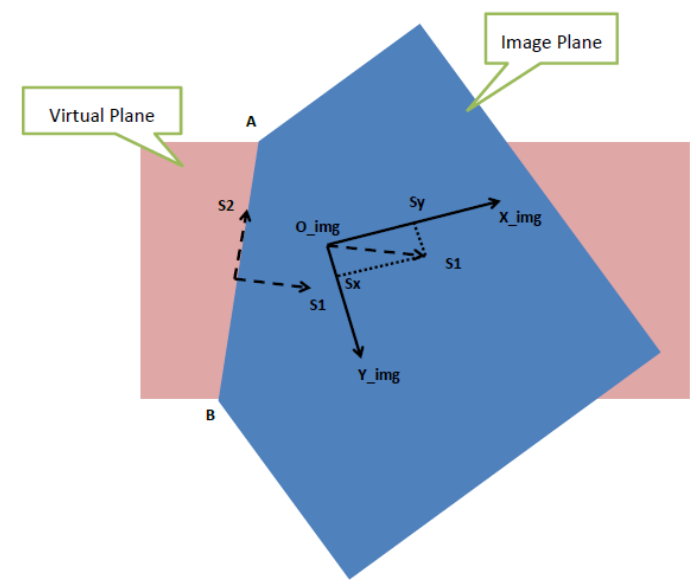

Fig. 2 Virtual plane and image plane intersect at line $A B$

The 3D relationship among these coordinate systems is shown in Fig. 3. 


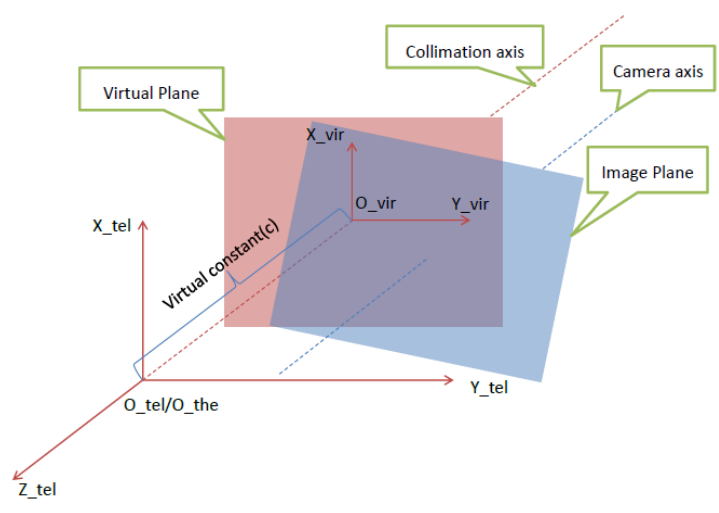

Fig. 3 Relationships among telescope coordinate system, virtual plane system and image plane system

The virtual constant should always be fixed because the shape on the virtual plane can change by a scalar factor with different virtual constants as shown in Fig. 4. We set $\mathrm{c}=10^{5} p x$ as this results in a scale factor near 1.00, which means that an one-pixel deviation on the image plane will lead approximately to one pixel deviation on the virtual plane. That helps to assess the accuracy of the estimated angles more conveniently.

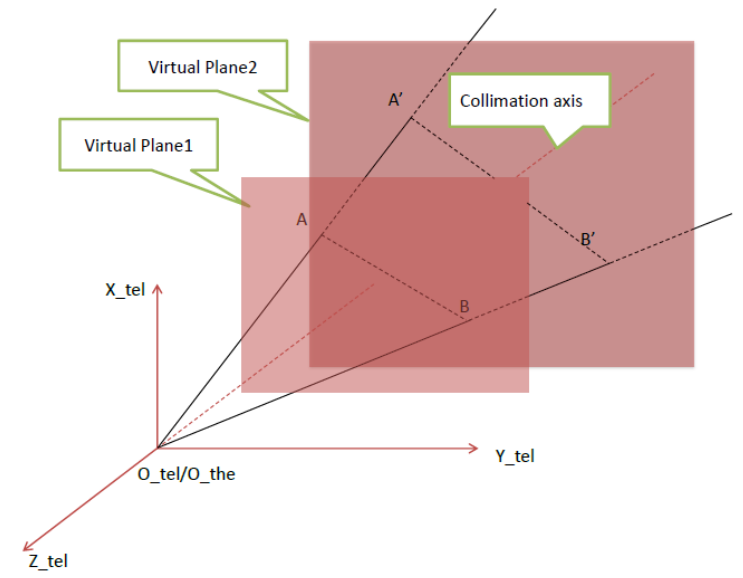

Fig. 4 Different sizes and positions of target in different virtual planes because of different virtual constants

The coordinates on the virtual plane are obtained from the image coordinates by 4 affine transformation parameters (Fig. 5). These 4 parameters $\left(a_{1}, a_{2}, b_{1}, b_{2}\right)$ are equivalent to those in Walser (2004) which may be expressed as $\mathrm{S}_{\mathrm{x}}, \mathrm{S}_{\mathrm{y}}$ for the scale factor in both directions, $s$ for shear factor and $\alpha$ for rotation angle.

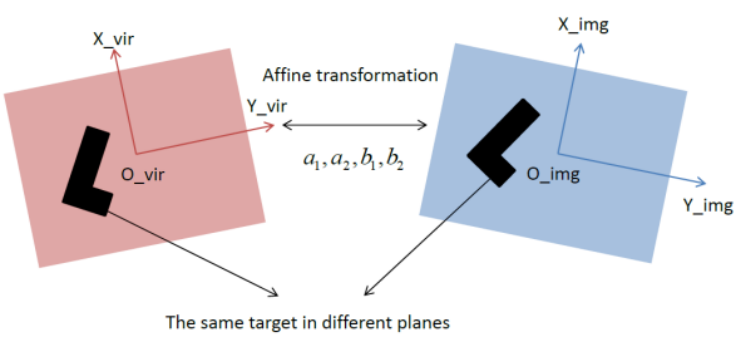

Fig. 5 Affine transformation between image coordinate system and virtual plane coordinate system

The equation is quite simple and easy for differentiation:

$$
\begin{aligned}
& x_{-} \text {tel }=a_{1} \cdot x I_{-} c+a_{2} \cdot y I_{-} c \\
& y_{-} t e l=b_{1} \cdot x I_{-} c+b_{2} \cdot y I_{-} c
\end{aligned}
$$

where $x \_t e l$ and $y_{-}$tel are telescope coordinates and have the same value in the virtual plane coordinate system.

For each target point we always get a 3D coordinate value $\left[\begin{array}{l}x_{-} t e l \\ \left.y_{-} t e l-c\right]\end{array}\right]$ in the telescope coordinate system, and we can transform it into the corresponding theodolite coordinate system by two rotation angles - the horizontal angle and the vertical angle.

For a leveled instrument, the theodolite $\mathrm{Z}$ axis should be pointing to the zenith direction and the $\mathrm{X}$ axis is pointing to initial zero direction. Since different instrument errors will always influence the angular measurement we need to compensate these instrument errors before using the angles to do the coordinate transformation. These errors are caused by the instrument itself and may be separated from the calibration parameters. We use the same mathematical model for calculating the theodolite axis errors in Walser (2004). The compensated horizontal and vertical angles are $H z_{-} t h C$ and $V_{-} t h C$. With the help of the crosshair in the telescope we can calculate the instrument errors before calibration. This is one of the major differences between Walser's method and ours. 


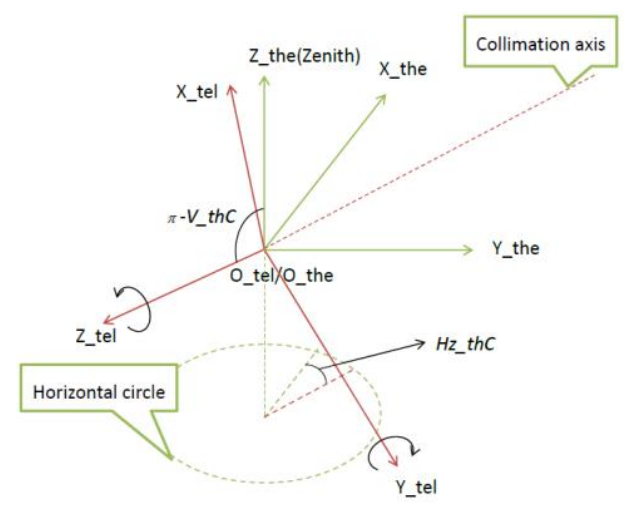

Fig. 6 Relationship between the theodolite coordinate and the telescope coordinate system

There are two steps involved in the 3D coordinate transformation from the telescope coordinate system to the theodolite coordinate system. The first step is to rotate $\pi-V_{-} t h C$ around Y_tel clockwise. Then rotate $H z \_t h C$ around $Z_{-}$tel counter clockwise. We have:

$$
\begin{aligned}
{\left[\begin{array}{c}
x_{-} t h e \\
y_{-} t h e \\
z_{-} t h e
\end{array}\right]=} & \boldsymbol{R z}\left(H_{-} t h C\right) \cdot \boldsymbol{R} \boldsymbol{y}\left(V_{-} t h C-\pi\right) \\
& \cdot\left[\begin{array}{c}
x_{-} t e l \\
y_{-} t e l \\
-c
\end{array}\right] \\
= & \boldsymbol{R} \cdot\left[\begin{array}{c}
x_{-} t e l \\
y_{-} t e l \\
-c
\end{array}\right]
\end{aligned}
$$

where $\boldsymbol{R} \boldsymbol{y}\left(V \_t h C-\pi\right), \boldsymbol{R} z(H z)$ and $\boldsymbol{R}$ are $3 \times 3$ rotation matrices and $\left[x_{-} \text {the } y_{-} \text {the } z_{-} \text {the }\right]^{T}$ are given in theodolite coordinate system.

If we define the positive direction of the rotation matrices as counter clockwise, we have:

$$
\begin{aligned}
\boldsymbol{R} y(\alpha) & =\left[\begin{array}{ccc}
\cos (\alpha) & 0 & \sin (\alpha) \\
0 & 1 & 0 \\
-\sin (\alpha) & 0 & \cos (\alpha)
\end{array}\right] \\
\boldsymbol{R z}(\alpha) & =\left[\begin{array}{ccc}
\cos (\alpha) & -\sin (\alpha) & 0 \\
\sin (\alpha) & \cos (\alpha) & 0 \\
0 & 0 & 1
\end{array}\right]
\end{aligned}
$$

where $\alpha$ is the rotation angle.

With the 3D theodolite coordinate obtained by Eq. (4), we can get the corresponding horizontal angle, vertical angle, and slope distance of the target. The slope distance is related with the virtual constant, but the angles are independent.

$$
\begin{aligned}
& H_{-} \text {tar }=\arctan \left(\frac{y_{-} t h e}{x_{-} t h e}\right) \\
& V_{-} \text {tar }=\frac{\pi}{2}-\arctan \left(\frac{z_{-} \text {the }}{\sqrt{x_{-} t h e^{2}+y_{-} t h e^{2}}}\right)
\end{aligned}
$$

Every time we point to the target direction within the FoV of the telescope camera, we can get the pixel coordinates of the target and the estimated horizontal and vertical angles of the target using the method above. The parameters to be estimated are:

$$
\hat{\boldsymbol{X}}=\left[\begin{array}{lllllll}
\hat{a}_{1} & \hat{a}_{2} & \hat{b}_{1} & \hat{b}_{2} & \hat{K}_{1} & \hat{H} z & \hat{V}
\end{array}\right]^{T}
$$

where $\hat{H} z$ and $\hat{V}$ are the estimated angles of the target in the theodolite coordinate system. The 4

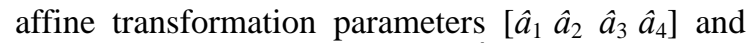
radial distortion parameter $\hat{K}_{1}$ determine the relationship between pixel coordinates and the corresponding angles of the target in theodolite system at a specific focus position, and the last two parameters $[\hat{H} z \hat{V}]$ are auxiliary parameters and not involved in real applications.

The position of the principle point is not included in the model because it is related to other parameters, which means that the design matrix is singular and the estimated principle point is not robust. The same effect is also described in Walser (2004). Thus, we fix the principal point at the center of the image, and the minor difference is absorbed by all the other parameters

Each point pair can establish two observation equations, so we need at least 4 point pairs to calculate the parameters. A least-squares adjustment method is used to estimate the variables with a unit weight matrix and the following initial values:

$$
\begin{aligned}
& a_{1}^{0}=1, a_{2}^{0}=0, b_{1}^{0}=0, b_{2}^{0}=1, K_{1}^{0}=0 \\
& x_{0}^{0}=1280 \text { pixel, } y_{0}^{0}=960 \text { pixel } \\
& H z^{0}=\text { mean }\left(H z_{-} \text {th } C\right) \\
& V^{0}=\text { mean }\left(V_{-} \text {th } C\right)
\end{aligned}
$$


We must be aware that the 5 parameters ahead will change when the focus position (i.e. the target distance) changes. As is mentioned in literatures, e.g. Bürki et al. (2010) or Wasmeier (2009), the calibration parameters are potentially subject to variations. Therefore, for each different focus position a separate set of calibration parameter has to be estimated. If enough parameter sets are determined (in our case there are 8 sets for the distance range between $2 \mathrm{~m}$ and $13 \mathrm{~m}$ ), we can interpolate the correct values at each possible focus position.

\section{Automatic Detection of Coded Targets and Multi-Target Monitoring}

Coded Targets detection is an edge-based measurement method. As is described in Reiterer and Wagner (2012), the edge-based method is the most precise detection method compared with template-based and point-based method. In Bürki et al. (2010), detection based on image processing algorithms has special benefits compared with Automated Target Recognition (ATR) functions. A detailed comparison is given in that paper, so we will not discuss it here again. For CTs, we can also get the ID of each target which makes the detection a fully automation procedure.

CTs have been used in photogrammetry for decades. In Ahn and Rauh (1998), different types of CTs were summarized and a circular CTs automatic detection was presented. The CTs we are using can be obtained by PhotoModeler or other photogrammetry software. After some basic processing such as Gauss filtering and image binarisation with a given threshold, we use the Canny algorithm (Canny 1986) to get the edges in an image. With all the edges, we can fit the corresponding ellipse boxes. These fundamental functions are involved in OpenCV libraries which are open and can be compiled in different platforms (Bradski 2000). CTs detection of concentric rings relies on two crucial steps. One is to correctly find the inner ellipse for positioning; the other is to obtain the robust and unique code in the outer code region for identification. As the CTs detection is already a mature technique and the detection procedure is not the topic of this paper, we will not discuss it in details here.

In monitoring applications using IATS and CTs, it is unnecessary to coincidently aim at the target with the cross hair. It is only necessary to keep the target point within the scope of FoV of the telescope camera. The FoV and the target point shown in
Fig. 7 are transformed from the image plane to the virtual plane by the previously estimated calibrated parameters. That FoV is actually a limited window scope in which the CTs can be precisely detected by automatic image detection. For each $\mathrm{CT}$ to be automatically monitored, there are several configuration parameters to be predefined, as shown in Tab. 1. Example images of CTs can be seen in Tab. 3.

Table 1. Configuration parameter for each monitored CTs

\begin{tabular}{ll}
\hline Symbol & Explanation \\
\hline$C$ & Code of the target \\
$H z$ & Horizontal angle of the collimation direction \\
$V$ & Vertical angle of the collimation direction \\
$F$ & Focus position \\
$G$ & Gray threshold for image binarisation \\
\hline
\end{tabular}

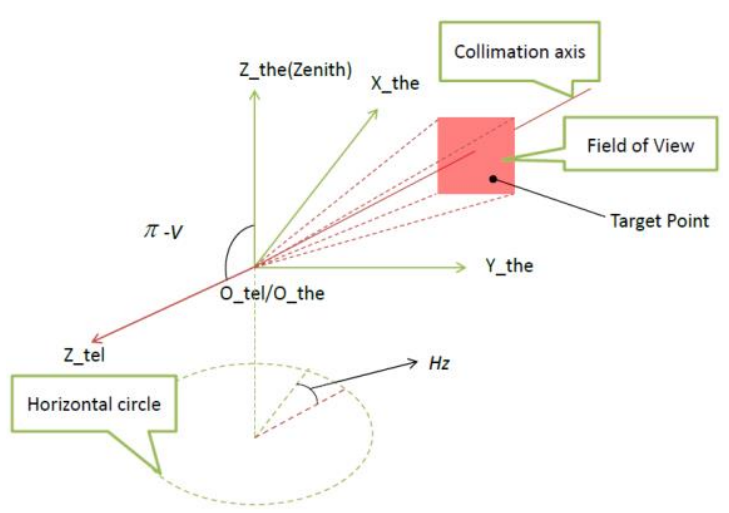

Fig.7 Field of view in the theodolite system

Every CT has a different unique code which represents an ID of the respective target. Different targets may have different positions and different distances to the total station, which means different focus positions to keep the shape of the CT on the image clear enough to be detected. Besides, the different CTs may be visible under different light conditions, so we have different input parameters to detect image coordinate of the target shape on the image. Here we use a threshold parameter for binarisation as the input parameter for image processing.

\section{Accuracy Assessment}

According to least-squares method, we have: 


$$
\begin{aligned}
& \boldsymbol{r e s}=\boldsymbol{A} \cdot \boldsymbol{\delta} \hat{\boldsymbol{x}}-\boldsymbol{l} \\
& {\left[\begin{array}{c}
r e s_{-} H z \\
r e s_{-} V
\end{array}\right]=\boldsymbol{A} \cdot \delta \hat{\boldsymbol{x}}-\left[\begin{array}{c}
\hat{H} z-H z_{-} \text {tar } \\
\hat{V}-V_{-} \text {tar }
\end{array}\right] \text { (9) }}
\end{aligned}
$$

where $\boldsymbol{r e s}$ is the residual vector of observations, $\boldsymbol{A}$ is linearized design matrix, $\delta \hat{\boldsymbol{x}}$ are corrections of variables using unit weight matrix. It's an iterative procedure with an initial value given by Eq. (8). The posterior variance of unit weight is:

$$
\hat{\sigma}_{0}^{2}=\frac{\boldsymbol{r e s}^{T} \cdot \boldsymbol{r e s}}{r}
$$

where $r$ is the degree of freedom. There are 7 variables to estimate in this method. If there are $n$ pairs of observations, we obtain $r=2 \times n-7$. The value of $\hat{\sigma_{0}}$ reflects the quality of original observations and how smoothly the original measurement fits the mathematical model.

From Eq. (1-6), we can see that there are two kinds of observations: one is the set of angular measurements obtained by the total station; the other is the set of detected pixel coordinates obtained by the telescope camera. The final result will be influenced by both measurements: the angular readings of the total station and the pixel coordinate detection of the CT on the image.

In our method, we use the virtual constant in the telescope system instead of the camera's principal distance in the camera system. One reason is that the principal distance changes when a different focus position is set, another is that we can directly obtain the angles in the theodolite system by this virtual constant and the image coordinates on the virtual plane. We set the virtual constant value as c $=10^{5} p x$ so that we can get a nearly identical size of the transformed image on the virtual plane compared to that on the image plane. Supposing the angular readings are ideally fixed, $1 / 10 p x$ deviation on virtual plane corresponds to 0.06 mgon in angles because of the distance of the virtual constant.

Due to the systematic effect or hysteresis effect, the angular readings will be a little different if we aim at the same target from different telescope positions. Wasmeier (2009) solves this problem by aiming at the target randomly, whereby this systematic error is averaged and applied to all the calibration parameters. To avoid this error, which will influence the accuracy of calibration result, we always need to preserve the same procedure when rotating the telescope to target direction. Fig. 8 shows how we rotate from left-top corner to the target $\mathrm{P}$ each time we do the measurement in calibration.

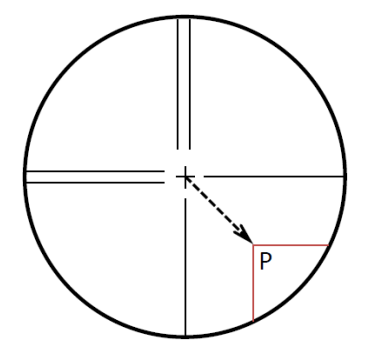

Fig. 8 The rotation direction of telescope to target $P$

\section{Calibration Experiment}

\subsection{Preparations}

The warming-up procedure is the first crucial preparation. The internal temperature can influence the output of angles and image point.

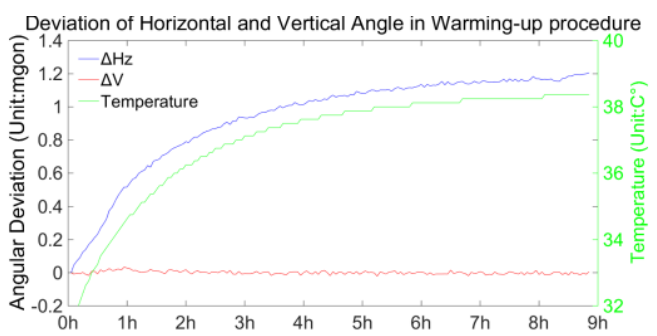

(a) Deviation of angles in warming-up procedure

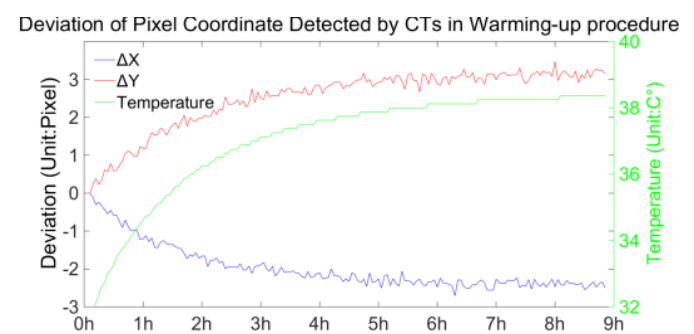

(b) Deviation of target pixel point in warming-up procedure

Fig. 9 Angle, pixel deviation and inner temperature in warming-up procedure: (a) Deviation of angles in warmingup procedure; (b) Deviation of target pixel point in warmingup procedure 
We can see from Fig. 9(a) that horizontal angle can deviate by up to 1.2 mgon while the vertical angle stays stable. Fig. 9(b) shows that the warming-up procedure can influence the CTs detection by $4.2 p x$ in the image, so this is only practical for long time monitoring. About 3 hours later the coordinate detection of CTs on the image stays stable. This long period is also verified by Wasmeier (2009) and Reiterer \& Wagner (2012). This fact must be considered by absolute measurements over longer periods. For short term relative applications it may be neglected.

Theodolite axis errors should also be considered before calibration. As is mentioned in Walser (2004), theodolite axis errors always occur when some axis conditions are not met. These errors include the vertical-index error, the collimation error, and the tilting-axis error, which can be determined and eliminated.

\subsection{Image Capturing and Corresponding Measurement}

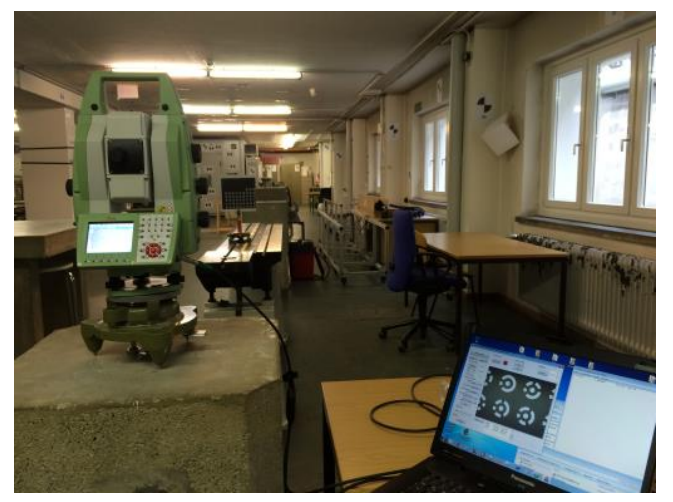

Fig. 10 Experiment at focus position 803

After the preparations mentioned above, we can start to obtain the calibration data. Here we use CTs as an example to detection the image position of a certain point. Other method such as cross line detection or simply the ellipse detection can also achieve the same goal. As described in Huang and Harley (1989) for the case of a single target, every time we rotate to a position close to the target, we can obtain a pair of angular readings and pixel coordinates of a detected CT. We have some amounts of observations that the fixed CT is distributed as a grid in the image. Fig. 10 shows one of the calibration positions with many CTs on the plate, and we only choose one of the CTs as our study target. As we use the coded targets only for calibration in a fixed set-up, an orthogonal view onto the target is guaranteed. In practical use case with a possible eccentricity error has to be considered, c.f. Luhmann (2014).

We have 16 pairs of aiming directions near our study target to estimate the calibration parameters for each focus position of the CT. Different focus position (and therefore different distances) of the CT means different parameters between the theodolite system and the image system. We obtained a total of 8 different focus positions. The relationship between focus position and distance to the CT is shown in Fig. 11.

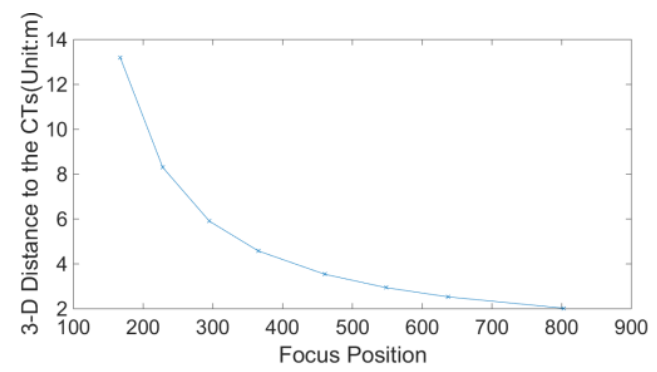

Fig. 11 Relationship between focus position and distance to the CT

\subsection{Results and Analysis}

According to the mathematical model and Eq. (10), we can estimate all calibration parameters and the accuracy of the result by using the least-squares method at different focus positions.

The results - as shown in Tab. 2 - are estimated based on equal weights. With these values, we can interpolate a new set of parameters for any focus position/target distance. As is described in Knoblach (2009), we can also establish polynomial functions between the focus position and these calibration parameters. More focus positions mean more precise sampling of the parameters for modeling. One thing needed to keep in mind is that the virtual constant shown in Fig. 3 should always be fixed both in the calibration and the application case. Different virtual constants will yield to different groups of calibration parameters by a scale factor. Similar with Bürki et al. (2010), $a_{1}$ and $b_{2}$ represent a change in the scales of the image. The graphical relationship between parameter $a_{1}$ and focus position is shown in Fig. 12. 
Table 2. Calculated results of 8 calibration parameter sets (focus positions)

\begin{tabular}{llllllll}
\hline $\begin{array}{l}\text { Focus } \\
\text { Position }\end{array}$ & $\begin{array}{l}3-\mathrm{D} \\
\text { Distance } \\
(m)\end{array}$ & $a_{1}$ & $a_{2}$ & $b_{1}$ & $b_{2}$ & $\begin{array}{l}K_{1} \\
\left(\times 10^{-5}\right)\end{array}$ & $\begin{array}{l}\hat{\sigma_{0}} \\
(\text { mgon })\end{array}$ \\
\hline 803 & 2.024 & 0.960227 & 0.002549 & -0.002687 & 0.960233 & -0.1178 & 0.07 \\
637 & 2.529 & 0.955317 & 0.002681 & -0.002352 & 0.955360 & -0.1133 & 0.06 \\
548 & 2.942 & 0.953194 & 0.002656 & -0.002442 & 0.953409 & -0.0973 & 0.06 \\
460 & 3.540 & 0.951768 & 0.002693 & -0.002554 & 0.952121 & -0.1000 & 0.04 \\
365 & 4.577 & 0.950740 & 0.002609 & -0.002516 & 0.951165 & -0.1059 & 0.06 \\
295 & 5.902 & 0.950507 & 0.002698 & -0.002715 & 0.951000 & -0.1119 & 0.05 \\
228 & 8.302 & 0.950601 & 0.002675 & -0.002524 & 0.950941 & -0.1169 & 0.08 \\
167 & 13.205 & 0.950772 & 0.002659 & -0.002573 & 0.950949 & -0.1072 & 0.07 \\
\hline
\end{tabular}

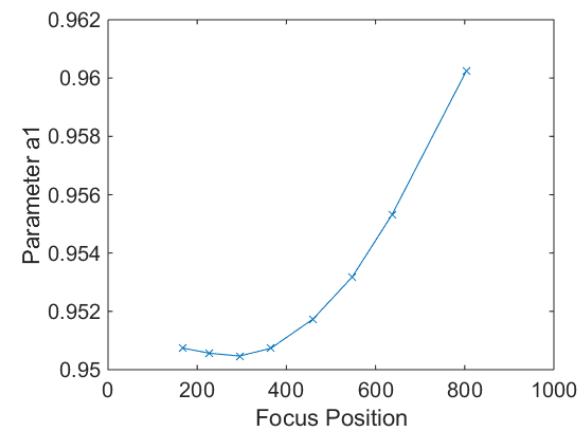

Fig. 12 Variation of the parameter $a_{1}$ in function of the focus position.

At each focus position, we have 16 image points distributed across the image. When the total station and the target are stably fixed, each pair of angular readings (horizontal and vertical angles) corresponds to a pair of image point position. Fig. 13 shows the angular residuals of the estimated angles in 16 directions to the same target using different calibration methods at the interpolated focus position of 508 .

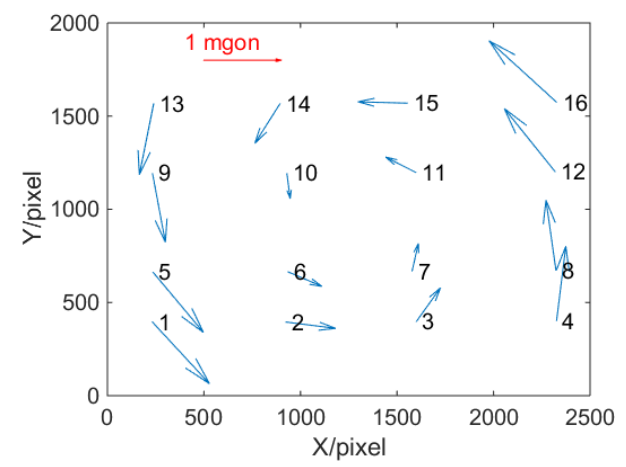

(a) Gnomonic projection

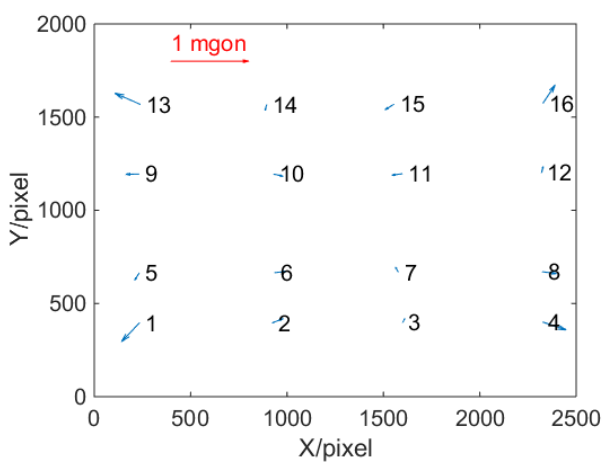

(b) Walser's method

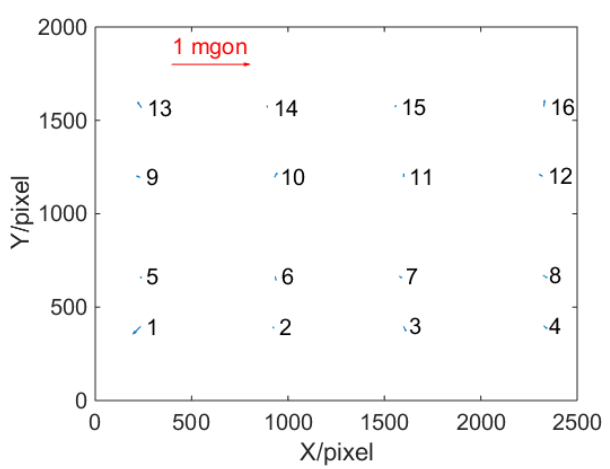

(c) Method in this paper

Fig. 13 Residuals of different calibration approaches using the same data at focus position 508: (a) Gnomonic projection; (b) Walser's method; (c) Method in this paper

Fig. 13(a) and Fig. 13(b) refers to the same calibration methods and parameters as described in Wagner and Wasmeier (2014), Fig. 13(c) shows the residuals calculated by this method. We use the same calibration data and obviously this method is the best solution for close range applications in our case. 
Gnomonic projection doesn't consider the rotation parameters and is only used for minor accuracy applications. As is described in Section 4, the rotation direction of telescope is important because different rotation direction to the same target will have different angular readings, which is also demonstrated in Fig. 14. Though the difference is quite small, it influences the calibration results. Walser's method doesn't take this point into consideration so the result can be influenced by random rotation direction of the telescope.

If we only use 4 affine parameters to calibrate, i.e. without the distortion parameter, and also aim at target from varying directions, we will get another set of 16 angular residuals which are not so good as in the method described in this paper. The differences are illustated in Fig. 14.

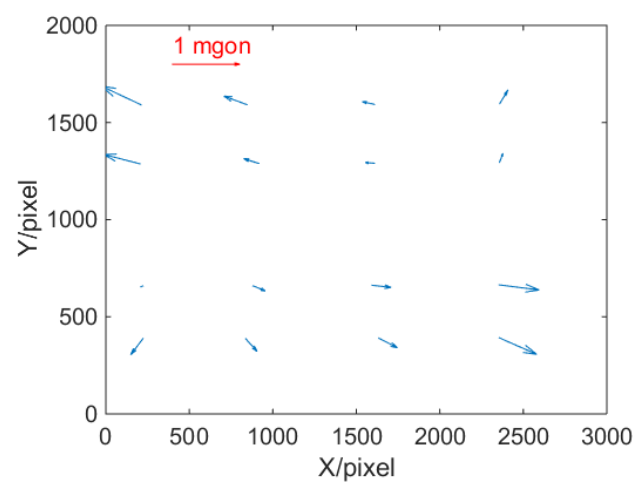

(a) Rotated in different directions and calibrated without distortion parameter

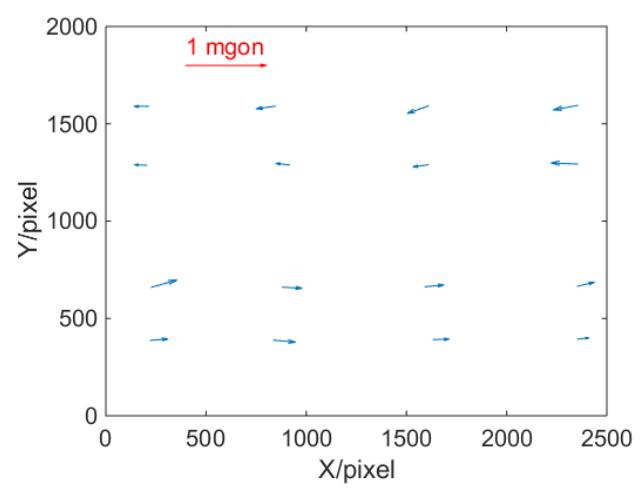

(b) Rotated in different directions and calibrated with distortion parameter

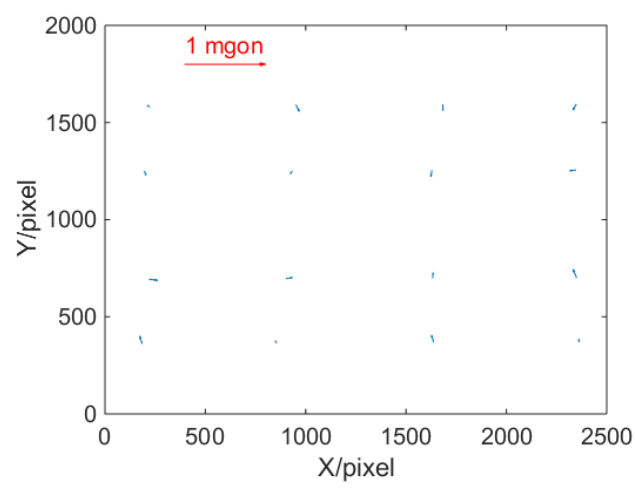

(c) Rotated in the same directions and calibrated with distortion parameter

Fig. 14 Residuals of different parameters and aiming directions: (a) Rotated in different directions and calibrated without distortion parameter; (b) Rotated in different directions and calibrated with distortion parameter; (c) Rotated in the same direction and calibrated with distortion parameter

Different rotation direction of the telescope will influence the angular readings of the total station (see the last paragraph in Section 4). The difference is quite small, but this factor exhibits a large influence on the calibration and cannot be ignored. The residuals shown in Fig. 14(a) are results of a calibration without distortion parameters and target aims in different directions. We rotate the telescope horizontal clockwise for the first two rows and horizontal anticlockwise for the last two rows. In Fig. 14(b), we also aim the target in opposite directions but calibrate with distortion parameters. In Fig. 14(c), we aim to the target always from the same direction and distortion parameter is involved in calibration. There are 5 parameters for transformation, and the maximum angular residual is only about $0.1 \mathrm{mgon}$.

\section{Multi-Target Monitoring Experiment}

Once the relationship between angular readings and pixel coordinates is determined at different focus positions, we can interpolate a group of calibration parameters for each new target distance. Further, this method provides a chance of replacing conventional ATR functions and can be used in various applications (e.g. automatic monitoring). Besides collimation angles (including horizontal and vertical angles) near the target prism in 
conventional automatic monitoring, the method in this paper involves more configuration parameters, as described before.

In this experiment, we choose 3 CTs indoors, which are attached on stable walls and foundations. The specific configuration parameters for monitoring are shown in Tab. 3 .

Table 3. Different configuration parameters for multi-target monitoring

\begin{tabular}{llrrrr}
\hline Image & $C$ & $H z$ (gon) & $V($ gon $)$ & $F$ & $G$ \\
\hline & 1683 & 186.76 & 98.88 & 211 & 178 \\
\hline
\end{tabular}

The gray threshold for image binarisation $G$ can be self-adjusted to a new value in the vicinity of input until the corresponding CTs can be successfully detected. For example, if the detection fails with the initial value $G=137$, the program can choose a new value like $G=142$ for detection. If still fails with $G=142$, it can choose $G=132$ for another detection. In the end when the detection succeeds, we can obtain a new $G$ value for the threshold. So this value is dynamically changed along with the light conditions.

The angle values $\mathrm{Hz}$ and $V$ value indicate that these $3 \mathrm{CTs}$ are located in different directions and focus position $\mathrm{F}$ indicates different distances from the targets to the total station.

In the monitoring procedure, every time we get a group of angular readings and pixel coordinate of the target on the image, we can calculate the corresponding angles in the theodolite system of the target based on Eq. (1-6) and the calibration parameters interpolated by focus position. The measured data of 3 CTs are shown in Fig. 15.
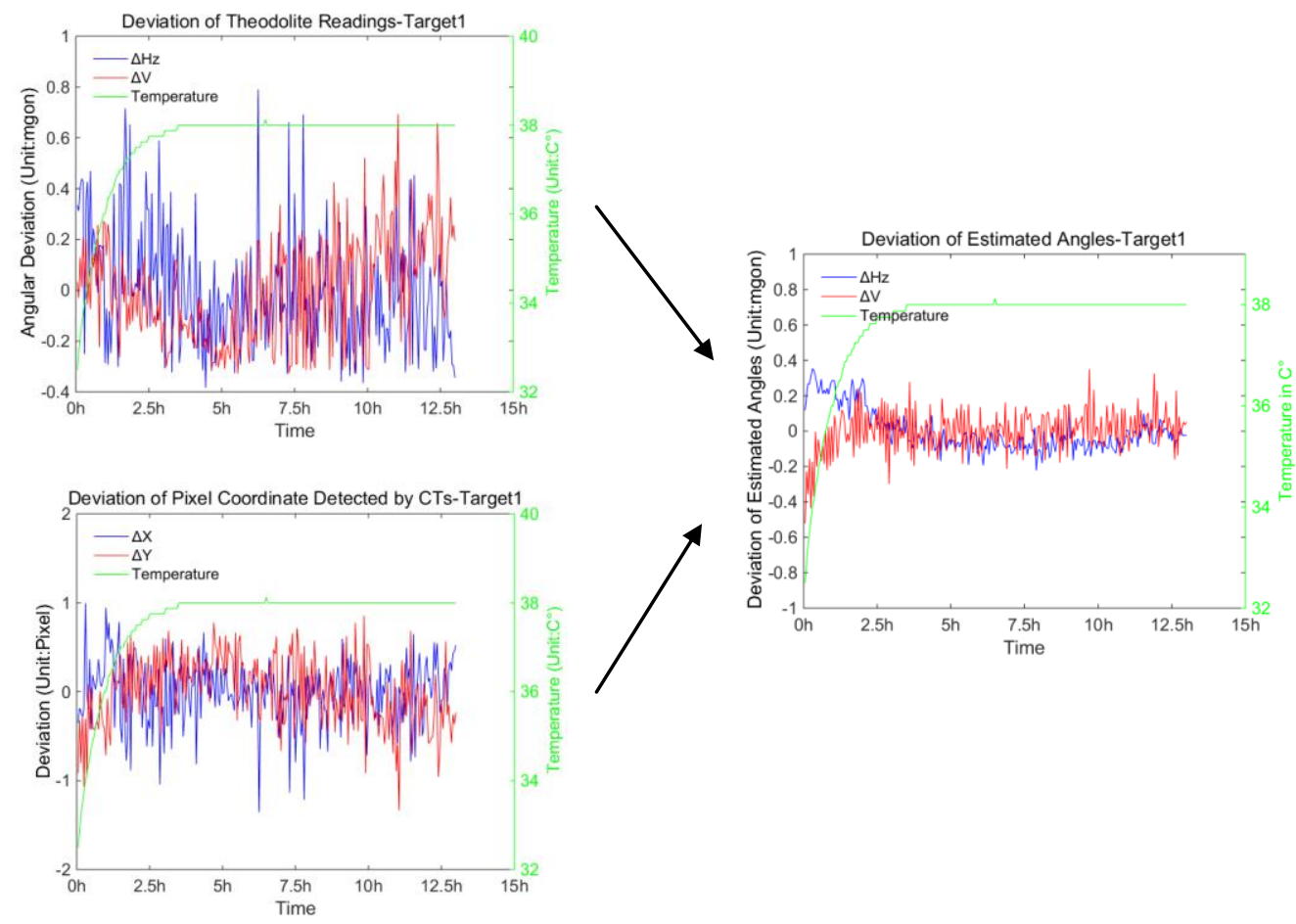

(a) Target 1 

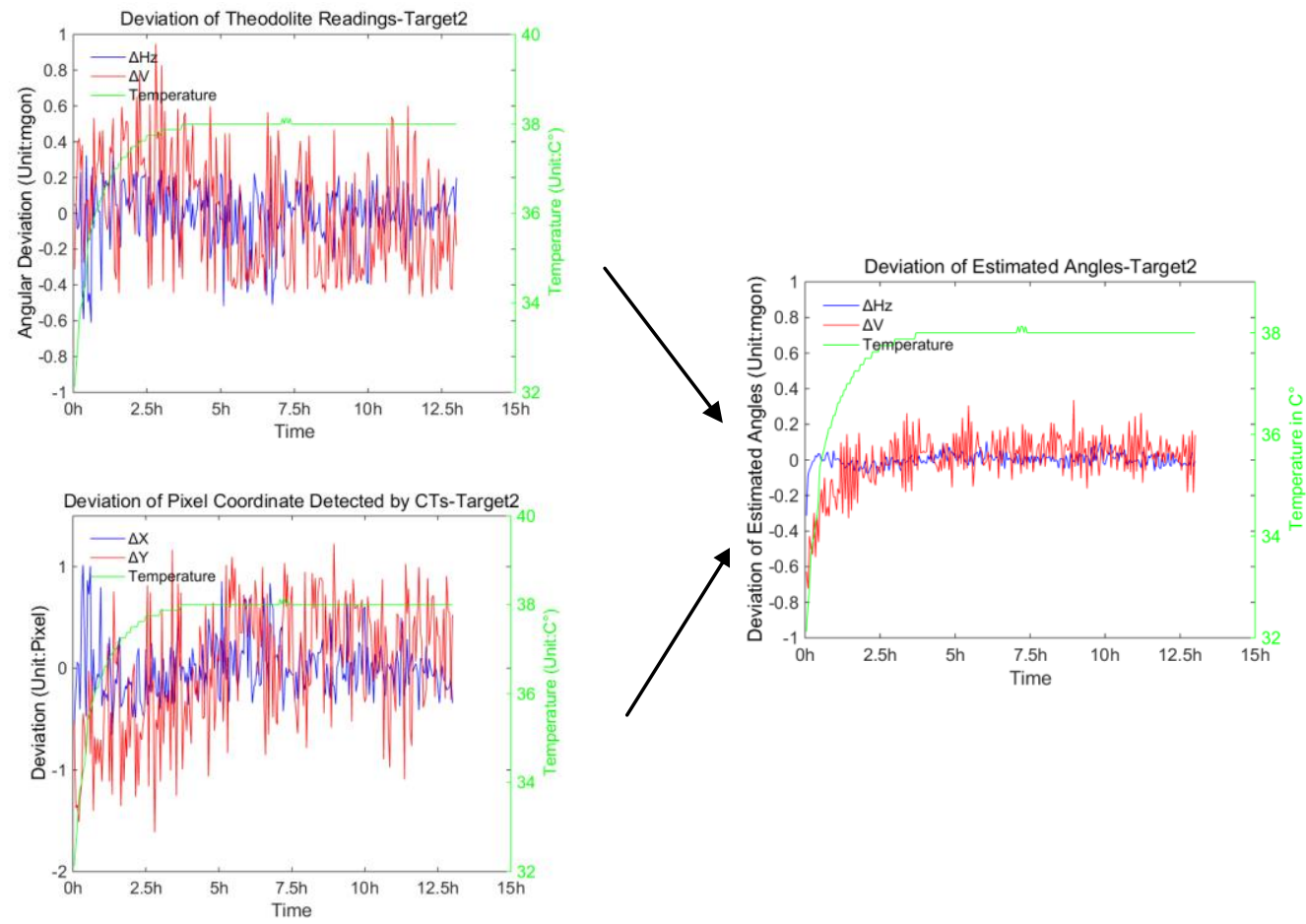

(b) Target 2
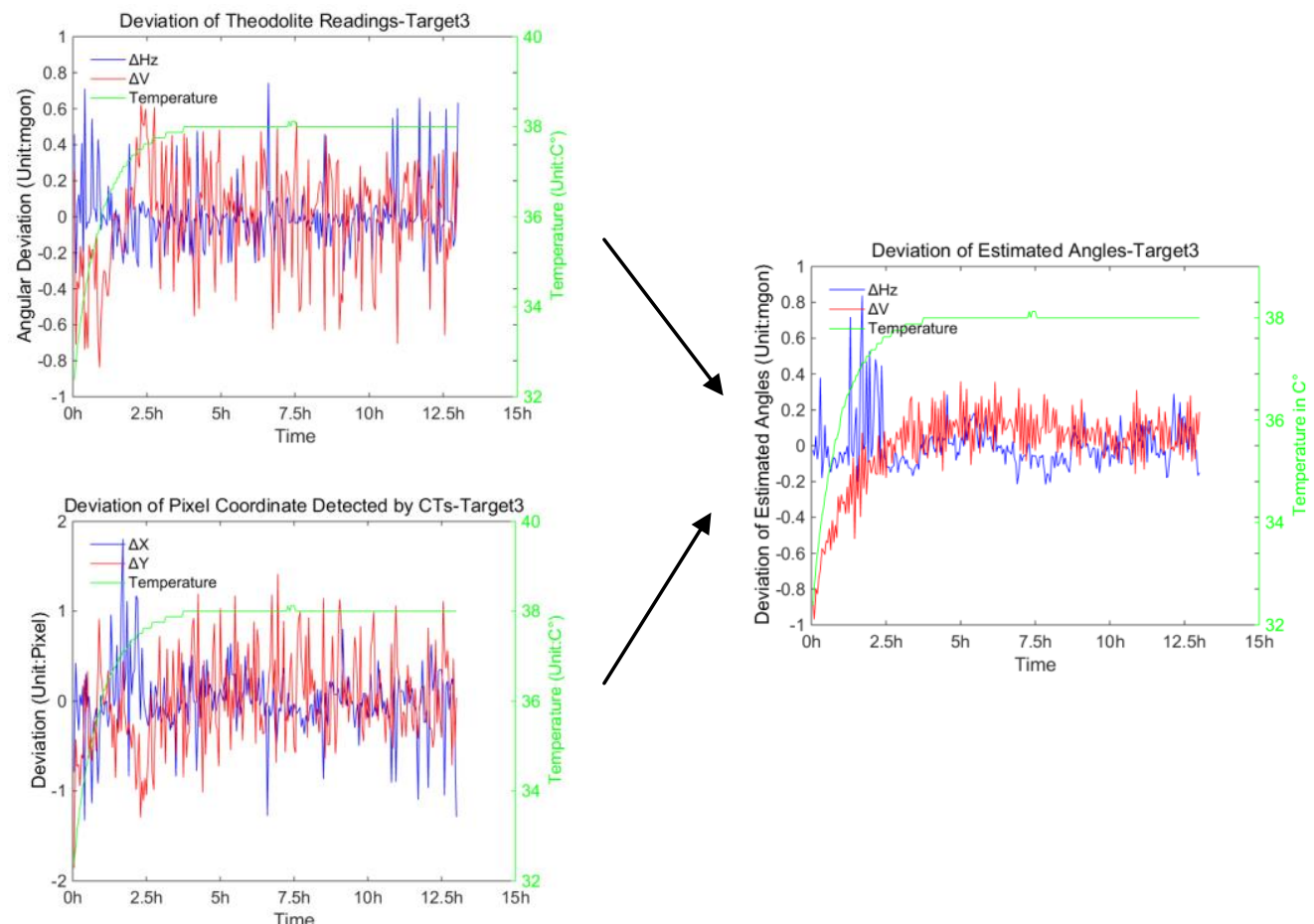

(c) Target 3

Fig. 15 Original monitoring data and the estimated angles: (a)Monitored data from target 1; (b)Monitored data from target 2; (c)Monitored data from target 3; 
The warming-up effect can also be seen in Fig. 15. One major difference is that the angular readings and detected pixel coordinates of the CTs look random and do not follow the same trend as in Fig. 9. It is because MS50 automatically rotates to a specific direction in each epoch, thus the accuracy of rotation itself should be taken into consideration. From Fig. 15, the original angular readings can deviate from approximately -0.8 to $0.8 \mathrm{mgon}$, even though we order the total station to position to exactly the same direction every time. But at the same time, the detected pixel coordinate of the target is analogously changed, which helps to yield a more accurate final result.

We choose and analyze the data after 3 hours of warming-up procedure and the result is shown in Tab. 4.

Table 4. Statistic results of the estimated angles after warming-up procedure (Unit: $m g o n$ )

\begin{tabular}{llll}
\hline \multirow{2}{*}{ Target ID } & Angle Type & $\begin{array}{l}\text { Max } \\
\text { Deviation }\end{array}$ & STD \\
\hline \multirow{2}{*}{ Target 1} & Horizontal & 0.16 & 0.06 \\
& Vertical & 0.34 & 0.10 \\
\multirow{2}{*}{ Target 2} & Horizontal & 0.10 & 0.03 \\
& Vertical & 0.29 & 0.10 \\
\multirow{2}{*}{ Target 3} & Horizontal & 0.30 & 0.09 \\
& Vertical & 0.28 & 0.11 \\
\hline
\end{tabular}

The last column STD in Tab. 4 stands for standard deviation. According to the description in the user specification of Leica MS50, the angular accuracy of GPR1 prism with ATR function is \pm 0.3 mgon. With this method we can get a comparable monitoring result to the conventional ways with cheaper cost.

\section{Conclusion and Expectation}

IATS is a promising development direction for total station. Many other image processing algorithms can be used and provide us a larger range of possible applications according to specific purpose. The presented method can replace the conventional monitoring method using the ATR function and prisms in some fields, especially because of its convenience and low-cost. The result can also be improved predictably by improving accuracy of the angular readings from total station and the target detection by image processing.

\section{Acknowledgment}

This paper is partly sponsored by China Science Council (No. 201406260148). We would like to appreciate two anonymous reviewers for their precious comments. We would also thank our colleagues Wolfgang Wiedemann and Max Hödel for their work supporting the preparation of this paper.

\section{References}

Ahn, S. J.; Rauh, W. (1998): Circular coded target and its application to optical 3D-measurement techniques. In: Proc. 20. DAGM Symp. Mustererkennung, pp. 245 -252.

Bradski, G. (2000): The OpenCV Library. Dr. Dobb's Journal of Software Tools.

Bürki, B.; Guillaume, S.; Sorber, P.; Oesch, H.S. (2010): DAEDALUS: A versatile usable digital clip-on measuring system for total stations. In: Indoor Positioning and Indoor Navigation (IPIN), 2010 International Conference on, Zurich, September 15-17, pp. 1-10.

Canny, J. (1986): A computational approach to edge detection. In: IEEE Trans. Pattern Analysis and Machine Intelligence, 8(6), pp. 679-698.

Devernay, F.; Faugeras, O. (2001): Straight lines have to be straight: automatic calibration and removal of distortion from scenes of structured environment. Machine Vision and Applications, 13(1), pp. 14-24.

Fitzgibbon, A.; Pilu, M.; Fisher, R.B. (1999): Direct least square fitting of ellipses. IEEE Trans. Pattern Analysis Machine Intelligence, 21(5), pp. 476-480.

Hattori, S.; Akimoto, K.; Fraser, C.; Ono, T.; Imoto, H. (2000): Design of coded targets and automated measurement procedures in industrial vision metrology. International Archives of Photogrammetry and Remote Sensing, Vol. XXXIII, Supplement B5.

Hattori, S.; Akimoto, K.; Fraser, C.; Imoto, H. (2002): Automated procedures with coded targets in industrial vision metrology. Photogrammetric Engineering \& Remote Sensing, 68(5), pp. 441-446.

Huang, Y.D.; Harley, I. (1989); A new camera calibration method needing no control field. Optical 3-D measurement techniques. Editors Gruen and Kahmen. Wichmann, Karlsruhe, pp. 49-56.

Knoblach, S. (2009): Entwicklung, Kalibrierung und Erprobung eines kameraunterstützten Hängetachymeters. $\mathrm{PhD}$ Thesis, Technische Universität Dresden.

Leica Geosystems AG. (2013). Leica MS50/TS50/TM50 - User Manual. User manual, v1.1.1, 84 pages.

Luhmann, T. (2014): Eccentricity in images of circular and spherical targets and its impact on spatial intersection. The Photogrammetric Record, 29(148), December 2014, pp. 417-433.

Reiterer, A.; Wagner, A. (2012): System consideration of an image assisted total station - evaluation and assessment. In: Allgemeine Vermessungsnachrichten (avn), 119(3), pp. 83-94. 
Tsai, R.Y. (1987): A versatile camera calibration technique for high accuracy 3D machine vision. International Journal of Robotics and Automation, 3(4), pp. 323-344.

Wagner, A.; Wasmeier, P. (2014): Flächen-und Featurebasiertes Monitoring mit Videotachymetern. MultiSensor-Systeme - Bewegte Zukunftsfelder. Schriftreihe des DVW, Vol. 75, pp.75-88.

Wagner, A. (2016): A new approach for geo-monitoring using modern total stations and RGB+D images. Measurement, Vol. 82, pp. 64-74.

Walser, B. (2004): Development and calibration of an image assisted total station. PhD Thesis, ETH-Zürich.

Wasmeier, P. (2009): Grundlagen der Deformationsbestimmung mit Messdaten bildgebender Tachymeter. $\mathrm{PhD}$ Thesis, Technische Universität München. 